\title{
Editorial: Evo-Devo of Color Pattern Formation
}

\author{
Ricardo Mallarino ${ }^{1 *}$, Marie Manceau ${ }^{2}$ and Marcus Kronforst ${ }^{3}$ \\ ${ }^{1}$ Department of Molecular Biology, Princeton University, Princeton, NJ, United States, ${ }^{2}$ Center for Interdisciplinary Research \\ in Biology, College de France, Paris, France, ${ }^{3}$ Department of Ecology and Evolution, University of Chicago, Chicago, IL, \\ United States
}

Keywords: evolution, development, pigmentation, Evo-Devo, color pattern formation

\section{Editorial on the Research Topic}

\section{Evo-Devo of Color Pattern Formation}

Because of their diversity, visual accessibility, and profound impact on the fitness of organisms in the wild, animal pigment patterns are one of the most fascinating traits found in nature. Although biologists have long studied the ultimate causes underlying pigment patterns (i.e., why they evolve) the molecular, genetic, and developmental mechanisms underlying their formation and evolution (i.e., how they are established) are less well-understood. In recent years, advances in genomic, molecular, and imaging approaches have opened an exciting opportunity to explore the mechanistic basis of color pattern formation in unprecedented detail.

Do the same genetic/developmental mechanisms underlie similar traits in different animals? Are the same/homologous genes repeatedly used by evolution to achieve the same purpose? What are the mechanisms by which gene regulator complexity is translated into phenotypic outputs? Unlike many other fields, in which the bulk of the research is predominantly carried out in a single species, researchers studying the mechanistic basis of color pattern formation capitalize on the strengths of a variety of model organisms, representing a wide taxonomic range. This approach provides an excellent framework for addressing long-standing questions in evolutionary developmental biology and gaining general insights into the molecular processes by which phenotypic diversity is generated.

This Research Topic on the evo-devo of color pattern formation brings together original research papers, perspectives, and reviews showcasing the wide variety of model systems (e.g., insects, reptiles, birds, amphibians, and mammals) and multidisciplinary approaches (e.g., experimental embryology, genomics, modeling, transcriptomics, imaging, etc.) that researchers are taking to study the mechanistic basis of color pattern formation. Below, we highlight the main findings of the contributions that form part of this fascinating Research Topic.

Specialty section:

This article was submitted to

Evolutionary Developmental Biology,

a section of the journal

Frontiers in Ecology and Evolution

Received: 18 June 2021

Accepted: 14 July 2021

Published: 06 August 2021

Citation:

Mallarino $R$, Manceau $M$ and Kronforst M (2021) Editorial: Evo-Devo

\section{INSECTS}

Butterfly wing pattern features known as "eyespots" have been well-characterized from the perspective of developmental genetics but we still lack a clear understanding of the function of eyespots or the cause of their divergence among species. Debat et al. study the evolution of eyespot number, size, and patterning across the neotropical butterfly genus Morpho (the big, flashy-blue butterflies). Interestingly, they identify traits, like eyespot number, that appear to evolve neutrally, and others, like position and size, that suggest selection.

Concealing mimicry is one of the most fascinating survival strategies: prey species evolve a color or shape resembling that of other species or their habitat, thereby deceiving predators. In this study, Yoda et al. describe a molecular interaction between products of pigment-producing genes in the control of butterfly pupae color dimorphism due to green or brown background matching. 
Hughes et al. investigate the gene regulatory networks (GRN) underlying dimorphic tergite pigmentation in Drosophila. By surveying an extensive number of fly species, they find that male-specific melanism is phylogenetically widespread and is established by the use of a common metabolic pathway. Interestingly, however, they find that the mechanisms underlying the regulation of this metabolic pathway differs among species, thereby indicating that there are different degrees of flexibility and constraint on GRNs.

Because of their marked differences in pigmentation patterns and powerful molecular tools, species in the genus Drosophila have provided fascinating insights into the genetic basis of phenotypic traits. Using state of the art genome editing tools, Lamb et al. demonstrate that the gene ebony, which had been previously found in a QTL mapping experiment, plays a major role in establishing pigmentation differences between two closely related fly species. In addition, they find that ebony affects cuticular hydrocarbon abundance in both species but does not control between species variation in this trait, demonstrating that cis-regulatory changes are able to cause divergence in some, but not all, traits affected by the same gene.

Lepidopteran color pattern diversity may seem boundless, yet through a detailed phenotypic survey of color distribution in 200 genera of butterflies, McKenna et al. identify common trends in four typical regions. By correlating these common themes with genetic marker distribution in imaginal disks, the authors show that positional information along the antero-posterior axis serves an important role in butterfly wing color pattern evolution.

The Odonota (Dragonflies and damselfies) display a considerable diversity of color patterns and have a visual system that is capable of recognizing such patterns, suggesting that this trait plays an important role in their ecology. In this review, Futahashi surveys the wide diversity of color patterns seen in this group and suggests a way of classifying them. In addition, he describes some of the physical and chemical properties that the bodies of members of this insect Order have evolved to produce such diversity of patterns.

The nymphalid ground plan (NGP), a theoretical archetype that describes characters in butterfly wings, has been widely adopted in comparative studies because it allows for the identification of homologous elements across species. Here, Gawne and Nijhout present a thorough analysis of the diverse wing patterns found in arctiid tiger moths and conclude that, while some of the wing elements of moths are present in the NGP, others are not. Based on these findings, the authors present a new theoretical groundplan that will provide a more comprehensive framework for comparative studies of Lepidopteran wing pattern evolution.

Wing patterns in Heliconius butterflies serve as emblematic study systems in evolutionary genetics and developmental biology. McMillan et al. provide here a thorough review of decades of work in this system to illustrate how recent progress opened new opportunities to answer long-standing questions on the spatio-temporal hierarchy of patterning signal propagation, the evolution of new regulatory variation, or the mechanisms constraining pattern diversification.
Developmental genes engrailed and invected, which trace back to an ancient gene duplication event, have both been implicated in various aspects of butterfly wing patterning. By studying a classic evo-devo model system, the butterfly Bicyclus anynana, Das Banerjee et al. find evidence for a third member of this gene family, which they name invected-like. They discover that these genes have diverged in their expression patterns, with engrailed being expressed in the posterior portion of the pupal wing as well as the eyespot center and a ring around the eyespot. Invected and invected-like, in contrast, are expressed only in the center of eyespots.

Wnt is a signaling pathway that participates in a myriad of developmental processes. Specific members of this pathway, like wingless and $W n t A$, have been shown to play a role in butterfly wing patterning. Fenner et al. explore the potential role of Wnt signaling on wing patterning in the butterfly family Pieridae, where little work has been done. They show that while WntA does not appear to be expressed during wing development, pharmacological treatment with heparin enhances melanization and alters UV reflectance, indicating a role for one or more Wnt genes during wing pattern development.

Shiny, metallic wing scales occur in a number of butterfly species but the exact structural features responsible for this trait remain a mystery. The unique appearance of these scales is known to be a product of their morphology, as opposed to pigments, but how do different butterfly clades produce these metallic scales and do they do it the same way? Ren et al. image metallic scales across five butterfly families and find some recurring themes as well as instances of unique scale morphologies in certain lineages. Their results suggest both similarities and differences in the origin of an apparently convergent phenotype.

Melanin is a central pigment across the animal kingdom and a core constituent of virtually all butterfly wing patterns. Furthermore, the biosynthetic pathway that produces melanin in insects is well-described. Kuwalekar et al. study the molecular evolution of six critical melanin pathway enzymes across Lepidoptera and show that these genes are largely shaped by purifying selection. Analysis of developmental expression patterns in one butterfly species, Papilio polytes, reveals marked instances of sexually dimorphic expression. Together, these results show how a core set of conserved genes can produce the diversity of lepidopteran wing patterns by altering the time and place of their expression.

\section{VERTEBRATES}

Domesticated animals, such as pigs, horses, and chickens, display a rich variety of coat and plumage patterns because these traits have been directly selected by breeders throughout many generations. In his review article, Andersson provides a detailed account of some of the mutations that disrupt existing color patterns or generate new ones. The examples illustrate the utility of using domesticated animals to map the genetic basis of traits.

While reviews of color pattern formation and evolution abound, none has thus far been dedicated to reptilian sauropsids 
(snakes, lizards) despite the extreme diversity in color, geometry, and ecological function of motifs adorning their skin. Kuriyama et al. fill this gap, linking current knowledge of pigmentation genetics and cell-based pigment production to theoretical and empirical work taking advantage of reptile color pattern attributes such as stripes and blue tails.

Cichlid fishes represent one of the classic examples of adaptive radiation and are widely known for their marked diversity in pigment patterns. Vertical bar patterns have independently evolved in multiple instances across the cichlid phylogeny but their developmental basis remains a mystery. By examining the ontogeny of vertical bar pattern formation in a cichlid species, Liang et al. provide a thorough account of the cellular basis underlying the formation of this trait and discover that it is primarily established by spatially localized changes in melanophore density, melanin synthesis, and melanophore dispersal.

Melanin-based coloration is associated with various behaviors and physiological traits. In this review, San Jose and Roulin discuss a breadth of recent work suggest that the link between these traits is their embryonic origin, namely the neural crest. Studying genes controlling the specification and differentiation of neural crest cell lineages for associated traits can thus provide a unique and powerful framework to integrate developmental biology and ecology.

Bird species have evolved a wide variety of pigment patterns, both across the body (macro) and within each feather (micro). In a review paper, Inaba and Chuong provide a thorough account of the cellular and developmental mechanisms by which macro- and micro-scale color patterns in birds are established and conclude that the marked differences seen in this group results from a complex interplay between tissues, cell types of different developmental origins, and interacting environmental factors.

Light skin color mutants are frequent in vertebrates: albino and leucistic phenotypes have been linked to genetic mutations in pigment production enzymes or pigment-cell differentiation. In this study, Ullate-Agote and Tzika show that leucistic Texas rat snakes lack melanophores and xantophores, but not iridophores. They pinpoint single nucleotide deletions in the coding sequence of MITF or decreases in MITF expression in leucisitic individuals, suggesting this gene controls the specification of neural-crest derived pigment-cells but not that of iridophores.

\section{EVO-DEVO THEORY}

Developmental genes are frequently thought to be expressed in a modular fashion, temporally and/or spatially, due to the action of modular cis-regulatory elements (CREs). This is a core concept in the field of evolutionary developmental biology (evo-devo). However, Lewis and Van Belleghem point out a number of examples in which CREs have been found to function in a pleiotropic, rather than modular, fashion. They propose "evolutionary modularity" as a potential explanation for how seemingly modular phenotypes might be controlled by nonmodular developmental genes.

\section{CONCLUDING REMARKS}

As evidenced by the contributions to this special issue, the evo-devo of color pattern formation is a thriving field, where researchers are employing a large variety of species and using multidisciplinary approaches to tackle a broad range of questions. Thanks to recent technical advances, the approaches and types of analyses that were once restricted to traditional model species are now widely applicable to a myriad of species with fascinating phenotypes. We anticipate that this trend will grow exponentially over the next few years, as researchers from more disciplines and backgrounds become engaged with the rich set of questions that remain wide open. By integrating the studies that are being done at multiple levels of biological organization-from molecules, to gene regulatory networks, to tissues and whole organisms-we will be able to obtain unprecedented mechanistic and conceptual insights into the processes that control color pattern formation and evolution.

\section{AUTHOR CONTRIBUTIONS}

RM, MM, and MK co-edited the Research Topic. All authors contributed to the article and approved the submitted version.

\section{FUNDING}

RM was supported by NIH grant: 5R35GM133758. MK was supported by: NIH grant GM131828 and NSF grant IOS-1922624.

Conflict of Interest: The authors declare that the research was conducted in the absence of any commercial or financial relationships that could be construed as a potential conflict of interest.

Publisher's Note: All claims expressed in this article are solely those of the authors and do not necessarily represent those of their affiliated organizations, or those of the publisher, the editors and the reviewers. Any product that may be evaluated in this article, or claim that may be made by its manufacturer, is not guaranteed or endorsed by the publisher.

Copyright (๑) 2021 Mallarino, Manceau and Kronforst. This is an open-access article distributed under the terms of the Creative Commons Attribution License (CC BY). The use, distribution or reproduction in other forums is permitted, provided the original author(s) and the copyright owner(s) are credited and that the original publication in this journal is cited, in accordance with accepted academic practice. No use, distribution or reproduction is permitted which does not comply with these terms. 